ए) ACUTE KIDNEY INJURY

\title{
SARS-CoV-2 in the kidney: bystander or culprit?
}

\author{
Anitha Vijayan and Benjamin D. Humphreys $\mathbb{B}$ \\ A new study examined post-mortem kidney tissue from 63 patients with \\ COVID-19. The results suggest that SARS-CoV-2 has kidney tropism, \\ including the ability to replicate in kidney cells, and that kidney transduction \\ by SARS-CoV-2 is associated with shorter survival time and increased \\ incidence of acute kidney injury. \\ Refers to Braun, F. et al. SARS-CoV-2 renal tropism associates with acute kidney injury. Lancet 396, 597-598 (2020).
}

Severe acute respiratory syndrome coronavirus 2 (SARS-CoV-2) emerged in late 2019 as a new virus causing severe respiratory illness and the COVID-19 syndrome. As the outbreak evolved into a worldwide pandemic, it became evident that infection with SARS-CoV-2 results in multi-organ disease, with a high incidence of acute kidney injury (AKI). Early reports from China did not describe the incidence and aetiology of AKI in COVID-19. However, subsequent studies from China, the USA and the UK report an incidence of AKI ranging from $17 \%$ to $43 \%$ in hospitalized patients. In critically ill patients, the incidence of AKI is remarkably higher, ranging from $61 \%$ to $76 \%{ }^{1}$. Now, Braun et al. report an association between SARS-CoV-2 infection of kidney cells and clinical outcomes in patients with COVID-19 (REF. ${ }^{2}$ ).

The exact pathophysiology of AKI in COVID-19 has not been clearly elucidated and is probably multifactorial (FIG. 1). As SARS-CoV-2 infection can lead to a severe systemic inflammatory response and microvascular thrombosis, AKI could be a result of haemodynamic fluctuations leading to ischaemic tubular injury. In one series from New Orleans, USA, AKI was attributed to ischaemic acute tubular injury in $66 \%$ of cases $^{3}$. The same researchers also reported that urine microscopy showed evidence of granular casts, which are characteristic of tubular injury, in $80 \%$ of patients. In addition, the onset of AKI in COVID-19 has been shown to closely correlate with timing of intubation, which is consistent with our own clinical experience (A.V. and B.D.H., unpublished work), suggesting that haemodynamic instability associated with mechanical ventilation could trigger ischaemic $\mathrm{AKI}^{4}$.

Although ischaemic and toxic acute tubular injury undoubtedly have a role in the aetiology of COVID-19-associated AKI, the very high incidence of AKI in this disease prompted speculation that direct SARS-CoV-2 transduction of tubular epithelial cells and perhaps podocytes contributes to kidney injury. This hypothesis was fuelled by the fact that angiotensin-converting enzyme 2 (ACE2), to which SARS-CoV-2 binds and which acts as the primary means of entry of the virus into the lung parenchyma, is highly expressed in the proximal kidney tubules ${ }^{5}$. Additional evidence suggesting possible kidney tropism of SARS-CoV-2 included multiple reports of collapsing focal segmental glomerulosclerosis (FSGS) in patients with COVID-19. In one series of six patients with COVID-19 (confirmed by positive PCR tests for SARS-CoV-2 from nasopharyngeal swabs), AKI and proteinuria who underwent kidney biopsy, kidney histology showed collapsing glomerulopathy and tubular injury ${ }^{6}$. Even though viral particles were not seen in the kidney tissue in this series, the manifestation of collapsing FSGS in COVID-19 is reminiscent of podocytopathies associated with other viruses such as HIV, parvovirus B19 (Parvo-19), BK virus and cytomegalovirus (CMV), where direct viral involvement has been documented.

The earliest evidence suggesting direct viral transduction of the kidneys in COVID-19
SARS-CoV-2 results in multi-organ disease, with a high incidence of acute kidney injury

came from a study that identified virus-like inclusions in tubular cells on electron microscopy in autopsy specimens ${ }^{7}$. Initially, this finding could not be replicated ${ }^{6}$ and putative SARS-CoV-2 particles seen by electron microscopy have been alternatively interpreted as clathrin-coated vesicles, microvesicular bodies or extruded microvesicles by other researchers ${ }^{8}$. In the first study to confirm the kidney tropism of SARS-CoV-2, investigators demonstrated viral RNA by in situ hybridization and PCR in all kidney compartments of autopsy specimens, most highly in glomeruli ${ }^{9}$. However, this study did not directly correlate the presence of viral RNA in the kidney with clinical characteristics.

The recent study by Braun et al. is the first to correlate SARS-CoV-2 kidney tropism with clinical outcomes ${ }^{2}$. The researchers collected post-mortem kidney tissue from 63 patients who had COVID-19 respiratory infection. It must be noted that to date, this cohort is the largest autopsy series to investigate SARS-CoV-2 and kidney tropism by a wide margin. Sixty per cent of the kidney samples contained SARS-CoV-2 mRNA. Patients whose kidney samples were SARS-CoV-2 positive were older, had more comorbidities and died sooner after SARS-CoV-2 diagnosis than patients with undetectable viral mRNA in their kidney. The presence or absence of AKI could be ascertained based on clinical data in 39 patients. Among those who developed AKI $(n=32)$, kidney SARS-CoV-2 mRNA could be detected in $72 \%(n=23)$, whereas in those without AKI $(n=7)$ viral mRNA could only be detected in $43 \%(n=3)$.

The presence of SARS-CoV-2 mRNA in post-mortem kidney specimens suggests that the virus can transduce kidney parenchyma, but does not provide direct evidence for such transduction. To address this important issue, the researchers homogenized kidney specimens to release SARS-CoV-2 viral particles and then incubated the homogenate with cultured primate kidney tubular epithelial cells. After 2 days of incubation, they could detect intracellular SARS-CoV-2 protein and a 


\section{$\int$ kidney tissue in patients with COVID-19 contains infective SARS-CoV-2 virus}

1,000 -fold increase in viral mRNA. This experiment provides definitive evidence that kidney tissue in patients with COVID-19 contains infective SARS-CoV-2 virus. Combined with the in situ and PCR data detecting SARS-CoV-2 in various kidney histological compartments, the strong implication is that SARS-CoV-2 can replicate in human kidney. However, formal proof of viral replication in human kidney cells remains to be shown.
This study represents an important step forwards in the rapidly growing body of evidence concerning SARS-CoV-2, kidney tropism and clinical outcomes. The recovery of viable SARS-CoV-2 from kidney provides the strongest evidence yet that the kidney is a target of the virus. One implication of this finding is that urine testing for viral mRNA might help to risk-stratify patients with COVID-19. The observation that SARS-CoV-2 kidney tropism is associated with premature death is consistent with the hypothesis that the virus might directly drive kidney injury. Chronic kidney disease has recently been identified as the strongest risk factor for COVID-19. related death - stronger even that chronic
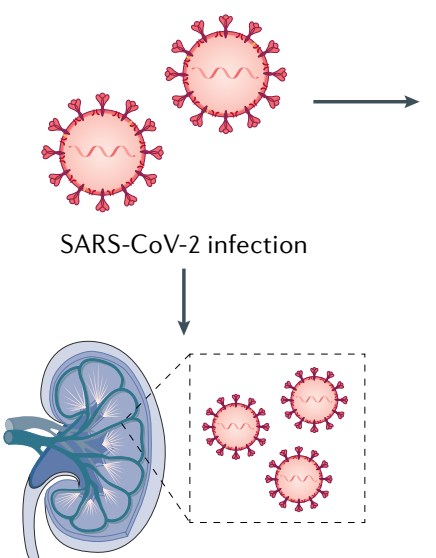

Viral transduction of kidney cells

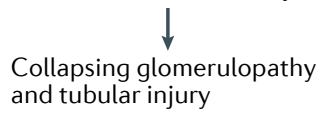
and tubular injury

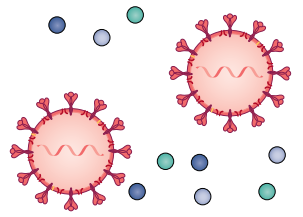

Systemic inflammation and
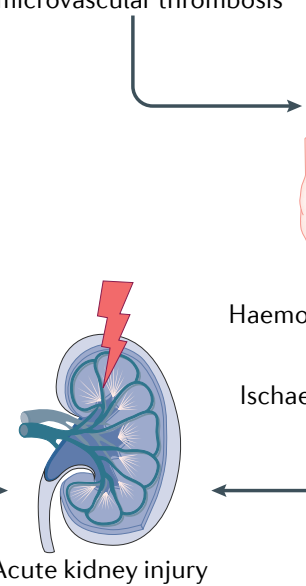

Acute kidney injury
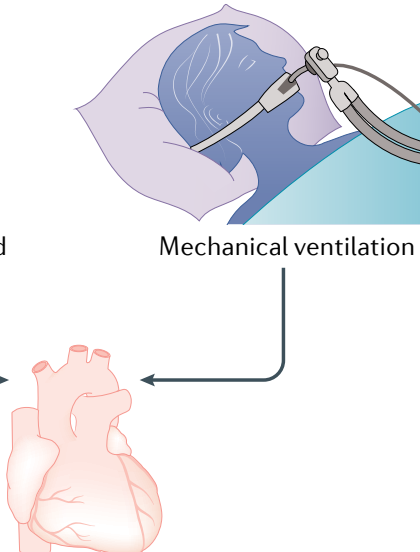

Mechanical ventilation

Haemodynamic instability

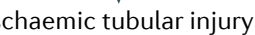

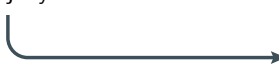

Fig. 1 | Potential mechanisms of AKI in COVID-19. SARS-CoV-2 may transduce podocytes, possibly leading to collapsing glomerulopathy. Alternatively, tubular epithelial transduction could lead to tubular injury and acute kidney injury (AKI). SARS-CoV-2 infection of lung parenchyma leads to systemic inflammation and microvascular thrombosis, contributing to haemodynamic instability. Periintubation hypotension may worsen kidney perfusion, leading to ischaemic tubular injury and AKI.

heart or lung disease - and one can speculate that this finding may also reflect SARS-CoV-2 kidney tropism ${ }^{10}$.

In summary, the study by Braun et al. provides important information regarding the kidney tropism of SARS-CoV-2 in COVID-19 and associates this tropism with disease severity. Future studies like this one are needed to better understand the cause of AKI in COVID-19 and to ultimately design effective therapeutic and kidney protective strategies.

\section{Anitha Vijayan ${ }^{\circledR}$ and Benjamin D. Humphreys (iD ${ }^{1,2}$ \\ 'Division of Nephrology, Department of Medicine, Washington University in St. Louis School of Medicine, St. Louis, MO, USA. \\ 2Department of Developmental Biology, Washington University in St. Louis School of Medicine, St. Louis, MO, USA \\ 凶e-mail: avijayan@wustl.edu; humphreysbd@wustl.edu https://doi.org/10.1038/s41581-020-00354-7}

1. Coca, S. G. et al. Acute kidney injury. NephJC http:/ www.nephjc.com/news/covidaki (2 August 2020).

2. Braun, F. et al. SARS-CoV-2 renal tropism associates with acute kidney injury. Lancet 396, 597-598 (2020).

3. Mohamed, M. M. B. et al. Acute kidney linjury associated with coronavirus disease 2019 in urban New Orleans. Kidney 360, 614-622 (2020).

4. Hirsch, J. S. et al. Acute kidney injury in patients hospitalized with COVID-19. Kidney Int. 98 , 209-218 (2020).

5. Batlle, D. et al. Acute kidney injury in COVID-19: emerging evidence of a distinct pathophysiology. J. Am. Soc. Nephrol. 31, 1380-1383 (2020).

6. $\mathrm{Wu}, \mathrm{H}$. et al. $\mathrm{AKI}$ and collapsing glomerulopathy associated with COVID-19 and APOL 1 high-risk genotype. J. Am. Soc. Nephrol. 31, 1688-1695 (2020).

7. Farkash, E. A., Wilson, A. M. \& Jentzen, J. M. Ultrastructural evidence for direct renal infection with SARS-CoV-2 J. J. Am. Soc. Nephrol. 31, 1683-1687 (2020).

8. Roufosse, C. et al. Electron microscopic investigations in COVID-19: not all crowns are coronas. Kidney Int. 98, 505-506 (2020).

9. Puelles, V. G. et al. Multiorgan and renal tropism of SARS-CoV-2. N Engl J Med 383, 590-592 (2020).

10. Williamson, E. J. et al. Factors associated with COVID-19-related death using OpenSAFELY. Nature 584, 430-436 (2020).

Competing interests

The authors declare no competing interests. 